\title{
Transdisciplinary Strategies of Discursive Practice: Procreation Effect
}

\author{
Larisa P. Kiyashchenko ${ }^{1}$ \\ 1 Institute of Philosophy RAS, Moscow, 109240, Russia
}

Received 14 September 2020 • Accepted 14 October 2020 • Published 31 December 2020

Citation: Kiyashchenko LP (2020) Transdisciplinary Strategies of Discursive Practice: Procreation Effect. Population and Economics 4(4): 67-73. https://doi.org/10.3897/popecon.4.e58665

\begin{abstract}
The paper aims at building a chain of semantic relations between the key concepts of the transdisciplinary discourse practices in relation to the procreation effect. To reveal the connections of the problem complex, the author provides a preliminary interpretation of concepts and semantic constructs forming a thematic thesaurus. Its key element is the concept of procreation, the definition of which derives its outline in the ways and rules of discursive practice, facilitating or hindering the impact of procreation on the process of normogenesis in the transdisciplinary perspective.
\end{abstract}

\section{Keywords}

transdisciplinarity; discursive practice; effect; normogenesis; procreation

JEL codes: J13

\section{Transdisciplinary Strategies}

Over the past 50 years, reference to interdisciplinary research has emerged in almost all areas of human activity focused on joint solution of problems that go beyond disciplinary restrictions on subject, methodology or language. Interdisciplinarity acts as the tool for harmonization of the languages of related disciplines, or for alignment of the languages of different and not necessarily close disciplines, and as a heuristic hypothesis-analogy, a constructive project, i.e., organized form of interaction of many disciplines for understanding, justifying and possibly managing the phenomena of supercomplex systems, and also as network, or self-organizing, communication (Budanov 2015: 75). Combined with interdisciplinarity, the transdisciplinary direction of research is also mentioned in the literature. Normally, transdisciplinarity is interpreted as a form 
of integration of, among other things, scientific knowledge, focused on the problems of the living world and assuming openness and digestibility of scientific information by the general public. And in such, one of the possible interpretations of transdisciplinarity, interdisciplinarity is seen as its forerunner. According to this approach, the need for transdisciplinary research arises in situations of complex socially significant problems, the solution of which can have a significant impact on the value landmarks of society as a whole or its individual groups (Kiyashchenko 2015). One cannot fail to notice the diversity of definitions of both interdisciplinarity and transdisciplinarity. And this is no coincidence, as both directions are young and are in the formative stage. Today many works are devoted to the analysis of their features. In general, it could be said that the specification of these directions depends on the choice of the research team, gathered by mutual interest to solve the problem of an urgent nature, with the necessary set of professional competencies, in the hope of contingent and prompt agreement on how to resolve it. Common to modern interdisciplinary and transdisciplinary research is the desire to find the lost unity in the perception of the world and ways to interact with it relying on the methods sometimes being developed right in the course of the research. The loss of unity in the relevant literature is often referred to as the crisis of European culture. The mentioned unity, as a universal value or a super task of today in natural sciences and humanities, is multiplied and fragmented depending on contextual (sociocultural) and local circumstances of the arising problem. But in the circumstances relevant today, the category of universality in its philosophical meaning takes on the paradoxical character of particular universality. And this trend of finding local unity is best manifested in transdisciplinarity. Transdisciplinary research in its fundamental meaning - the desire to move beyond the boundaries of interdisciplinarity into a transcending metaposition, enables numerous things, such as: understanding the complexity of the problem as a whole, taking into account the diversity of events of the living world and the possibility of scientific consideration of the problem, connecting abstract and specific practical knowledge, developing knowledge and activities involving achievement of the common good through an approximating approach to it in a particular life situation. Transdisciplinary research, which is based on an ethical project focus, is essentially a special experience of conducting a study that can be said to be reformatting its classic examples.

The practical orientation of the transdisciplinary strategy contains an impetus to the need for ethical justification and self-justification of decision-making and actions in human activity. This ability and possibility is rooted in the nature of human focused on the dual reproduction of oneself - on the one hand, as a bodily, psychophysical being represented in self-description practices in the language of ordinary representations, and on the other, as a creature that can go beyond the given circumstances of being, look at oneself and at the whole semantic texture distantly, which is characteristic of the philosophical approach. These conditions of transdisciplinary orientation can be considered independently of each other up to a certain point, but at the same time one should bear in mind their principled procedural relationship. The focus of the transdisciplinary approach brings together philosophical, theoretical and practical flows aimed at solving the arising problem. For this reason, the concept of transdisciplinarity has not only an applied aspect, but also a fundamentally existential character, significant to all those who professionally or by belief, by faith and by their life principles make choice in favour of a responsible, moral act (Kiyashchenko et al. 2009). 
Transdisciplinarity belongs to those modern areas of scientific research, in which, using the expression of Jürgen Habermas (Habermas 2000: 26), "a philosophical element within the sciences is distinctly expressed". This philosophical element (1) is focused on the philosophical clarification of the single, universal bases of experience of transdisciplinarity and phenomena of the surrounding world, and (2) contains issues that are resolved in specific ways of interaction in their complex diversity of disciplinary fields (physics, chemistry, biology, psychology, sociology). At the same time, the obtained solvings go beyond disciplinary scope and gain transdisciplinary significance.

In the understanding the above-mentioned transdiciplinary generality, the position of the philosophy of transdisciplinarity is also close to the ideas of Habermas (Habermas 2002: 21), who argued that the meaningful comprehension of the arising existential and philosophical problems is somehow connected with the scientific and philosophical mind coming close to the borders with the living world. The structures of the living world begin to perform the functions that fundamental philosophy used to claim, i.e., the function of an entity uniting the world or interconnecting life experience.

This metaposition of the transdisciplinary strategy is similar to the action of philosophical reflection, which, as a form of complex reflexive analysis of existence associated with relevant terminology, is certainly distant from common sense and ordinary consciousness, but nevertheless bonded to this ordinary consciousness with invisible threads, always acting as a form of deep personal consciousness. This is not just a collection of knowledge, but also personal experience of the world, which means that there is always some projection on the world of individual consciousness (Knyazeva 2018: 7, 29). Thus, a transdisciplinary strategy, acquiring in addition to the disciplinary an existential dimension, in one way or another expressing the intentional focus of consciousness on the object (thing), acquires the ability to act as an intermediary. Mediation is another term that is given to a thing, relative to the thing, in other words, both "inside" and "externally". "Only by projecting oneself onto the way of being of a thing does one become the "middle" between the infinite and the finite, outlining the ontological dimension of things, that is, that they are the essence of synthesis of presence and meaning. In this regard, one can say that a thing is a thing only if it can present itself and be reprimanded if it can affect me in my finiteness and be prepared for reasonable discourse" (Ogurtsov 1993: 18-19). This is what, according to Paul Ricoeur, forms philosophical anthropology," the infinite-finite driven by polarity (Ogurtsov 1993: 19). The specified polarity is antinomic in nature, i.e., in other words, its constituents exist "inseparably and distinctly" in its form. Other conceptual polarities of the philosophical discourse act in this capacity: general - unique, abstract - specific, fundamental - applied and collective - personal, etc., forming a mobile conceptual configuration of the problematic field in which modern human exists, acts, reflects, and discusses in the communicative space that arose on the basis of commonality of interests. The projection of individual consciousness on the world, which was referred to above, "senses" the previously established boundary with it or the newly formed here and now by the means available to the subject (knowledge, practical actions). The known expression "blind man's cane", rests either in paradoxical combinations of conceptual polarities or in the antinomic with the previously known, in other words, to the radically different, which makes thematization of reflexive subjectivity possible as free and responsible for achieving the truth (Ricoeur 2017: 82). The meaning of the latter is conventionally certified when meeting with communicants thinking differently about the same thing through the resolution of the apories of discourse (Mironov 2015). 


\section{Discursive practice}

To clarify the meaning of discursive practice used in our context, we will indicate how we see it. It consists, firstly, of the concept of discourse and, secondly, of the concept of discourse analysis. To date, there is a wide variety of their interpretations contained in numerous academic works. Systematization and bringing this diversity to a common foundation is a matter of the future, if it is, anyhow, possible. However, it is obvious that there is a great demand for these concepts. Discourse, discourse analysis and, finally, discursive practice, as a unifying concept, were formed at the end of the last century within the framework of social constructionism. These terms reflect a theoretical understanding of communication processes and their practical embodiment, and have proved to be in demand not only in linguistics, but also in other social sciences focused on solving interdisciplinary problems.

So, if discourse is a verbally articulated form of objectification of the content of human consciousness which is regulated by the type of rationality dominant in a particular sociocultural tradition, and discourse analysis is a set of analytical methods of interpretation of various kinds of texts or statements as products of verbal activity carried out in specific socio-political circumstances and cultural and historical conditions (https://gtmarket.ru/), then discoursive practice, which combines the meanings of the discourse and discourse anal$y$ sis concepts, unfolds in the space of communicative interaction when solving interdisciplinary and transdisciplinary problems in a conversational mode. Discursive practice is both a method of cognition and a creative act. In other words, it is responsible for carrying out an integrated transdisciplinary experience in which activities are to a certain extent proceduralised in a disciplined manner and at the same time forced to withdraw beyond normative and empirical limits. Existential experience of transdisciplinarity can be described from the point of view of the special mood of communication participants, of the mood for opposition to the interaction of the universal and the special. Here arises a new type of experience, when the universal participating in it is transferred to the private cases of personal special existence, and the latter "glow" with the code of the universal and holistic. At the same time, "the aspects of things most important to us, - Ludwig Wittgenstein wrote, - are concealed because of their simplicity and mundaneness. (They are not noticable because they are always before one's eyes). In other words, what we do not notice [up to a certain moment], having been seen once, turns out to be the most exciting and strong" (Budanov 2015: 129). This setting ("background" character and "revealing" ability of practices) is inherent in most studies within the practical paradigm (Issers 2011: 230). At the same time, on a case-by-case basis, the various fragments of practices adopted in a given culture function as knowledge of how to treat people and objects in order to achieve certain goals (ibid.). Practices are not fundamentally concealed, but a certain technique of description and interpretation is required to consider them. Thus, it can be concluded that the understanding of discoursive practice, like any social practice, is based on its understanding in the role of activity and social experience given in the direct observation (Issers 2011: 231). Let us emphasize the important topic for further presentation, namely, that in discoursive practice there is necessarily a descriptive part of the observed action, and its comprehension, from a detached position from beyond, the meta-position, observing the action taking place from the sidelines. Both have their own peculiarities and rules of carrying out, which "unfusedly" but "inseparably" constitute the complex unity of discursive practice, which in its course creates its own rules and conditions for their implementation - the meaning and norms of a particular type of practical activity. 
Thus, moving from the background topic setting in the concept of "transdisciplinary strategies" through reference to the fundamental move of conducting these strategies - discursive practice, we stated the combination of linguistic realities (rules and norms) of everyday language of description (discourse) and conceptual nomenclature in subjective (reflexive) execution of existing rules and norms (discourse analysis). It was noted that updating the creative potential of discursive practice is possible through overcoming (resolving) obstacles (antinomies, paradoxes, failures in understanding communication) at the boundaries of interaction of everyday language describing a private case with a reflexive assessment of its vision as a whole in the integrated perspective of the transdisciplinary approach and its norms. It can be said, in a known approximation, that normogenesis is the epicenter that dynamizes transdisciplinary study.

It is observed that increasing complexity is based on dissipation and increasing chaos. This is the paradoxical meaning of Prigozhin's term dissipative structure: the ordered structure arises not in spite of, but due to dissipation. The tendency of increasingly complex structures to emerge in the course of evolution and self-organization is associated with increasing creativeness, the birth of innovation, which some scholars call the order for freedom (Knyazeva 2018).

\section{Normogenesis: procreation effect}

Now, we have approached the key concept of our research - procreation. It is known that the scope of application of this concept is related to procreation, reproduction of offspring, a specific direction of human activity in the field of medical care, not devoid of sociohumanitarian attributes. However, the use of this concept in a broader context, as a process and result of sociocultural normogenesis, makes it more fundamental, applicable to other areas of human activity and testifies to the metaphorical nature of the concept of procreation. With this approach, occur a shift and enrichment of the meaning of the concept, and this does not abolish its use in the medical field, where it originates from. Procreation, as a living metaphor (Ricoeur 2015), accumulates interdisciplinary differences in dispute with everyday language of communication between the normative order of new technologies, the logic of scientific, medical, and sociological calculations and search new meanings of procreation, parenthood, kinship, and family values in an ethico-axiological way. Metaphors create a "shimmering zone" of the origin of meaning: an area where different fields of knowledge that were previously considered unrelated can find contact. In these areas, when connecting previously unrelated knowledge, it is possible to give rise to completely new ideas in any field of science and culture (Arutyunova 1990). And these are areas of renewal and formation of norms and regulatives of behaviour where the process of interaction of spontaneity and consciousness occurs. The objective process of formation of norms, which is always, and in all societies, spontaneous, must be distinguished from the subjective consciousnessly focused process of their formulation (orally or written) by relevant authorities. Consideration of the concept of procreation as a living metaphor linking the fundamental and applied levels of human activity gives a chance of conceptual synthesis of fundamental ideas in comprehension of modern human reproduction processes in the context of their biotechnological transformation, and the results of applied research of these processes in sociological, medical, cultural, anthropological, and psychological aspects in interdisciplinary discourses, which can be based on the formation of practical philosophy, namely, the 
assumption that language and conceptual constructions of discursive practice can become the basis of combining different levels of ways of describing transformations in human reproduction and sociocultural practices of reproduction, which are expressed in disciplinary discourses focused on solving acute existential problems (at the interface of personal formation and physical health).

\section{Conclusion}

Any scientific study, understood as a communicative process, is a complex variety of cognitive acts includes questioning and anticipation of the answer, consent and objection to other communication participants. The cutting edge of science is the field of interaction of many fundamentally equal consciousnesses, in which there is a divergent, differentiated agreement. Science, understood as interference of acts of communication, is subject to certain norms and patterns of interaction of scientists. These norms and samples, which ensure the stability of scientific knowledge, are deposed in the system of disciplinary knowledge and in certain ideals and criteria of scientific knowledge identified by the methodology of science (Neretina et al. 2017). The stability of the resulting invariant is relative. It is "washed" by the multiple variability of divergent discoursive practice focused on its renewal in accordance with the strategies of transdisciplinary research.

Acknowledgements. The research was carried out with the financial support of RFBR within the framework of scientific project No. 20-011-00609

\section{Reference list}

Arutyunova ND (1990) Metaphor. Linguistic encyclopedic dictionary. Soviet Encyclopedia, Moscow, 296-297. (in Russian)

Budanov VG (2015) Interdisciplinarity and transdisciplinarity of the early 21 st century. Philosophy, Methodology and History of Science 1: 100-112. (in Russian)

Habermas J (2000) Moral consciousness and communicative action. Saint-Petersburg. 380 pp. (in Russian)

Habermas J (2000) The future of human nature. On the way to liberal eugenics? Moscow 144 pp. (in Russian)

Issers OS (2011) Discursive practice as an observable reality. Omsk University Bulletin 4: 227-232. (in Russian)

Kiyashchenko LP (2015) Philosophy of transdisciplinarity: approaches to definition. In: Transdisciplinarity in philosophy and science: approaches, problems, prospects. Publishing house "Navigator", Moscow, 109-135. (in Russian)

Kiyashchenko LP, et al. (2009) Philosophy of Transdisciplinarity. IFRAN, Moscow. (in Russian)

Knyazeva EN (2018) Biosemiotics: the origins of the interdisciplinary direction. Issues of Philosophy 11: 89-98. (in Russian)

Mironov VV (2015) On the peculiarities of philosophical reflection and semantic space. Philosophy, Methodology and History of Science 1(1): 28-37. (in Russian)

Neretina SS, Ogurtsov AP, Murzin NN, Pavlov-Pinus KA (2017) Apories of Discourse - Joint monography. Chief editor S.S Neretina. Institute of Philosophy of RAS, Moscow, 119 pp. (in Russian) 
Ogurtsov AP (1993) Scientific discourse: power and communication (complementarity of two traditions). Philosophical Studies 3: 12-59. (in Russian)

Ricoeur P (2015) A living metaphor. Seventh Essay: Metaphor and Reference. Horizon 4(1): 175-213. (in Russian)

Ricoeur P (2017) Philosophical Anthropology. Manuscripts and speeches 3 / Transl. from Fr. Humanitarian literature publishing, Moscow, 312 pp. (in Russian)

Shemanov AYu (2008) Reflectivity of cultural forms and problems of education. MSIC Bulletin 6: 82. (in Russian)

Wittgenstein L (1994) Philosophical Research - Philosophical works. P. 1. Gnozis, Moscow. (in Russian)

\section{Information about the author}

- Larisa Pavlovna Kiyashchenko - Doctor of Sci. (Philosophy), Leading Researcher at Institute of Philosophy of the Russian Academy of Sciences. E-mail: larisakiyashchenko@ gmail.com 